\title{
Adiabatic rotation of effective spin. II. Spin-rotational relaxation
}

\author{
Yu. A. Serebrennikov and U. E. Steiner \\ Fakultät für Chemic, Universität Konstanz, D-78434 Konstanz, Germany
}

(Received 1 November 1993; accepted 8 February 1994)

The theory of electron spin-rotational (SR) relaxation in systems with an effective spin $S^{\text {eff }}=\frac{1}{2}$ is formulated in terms of the adiabatic rotation of effective spin (ARES) approach. It is shown that SR relaxation results from the orientational random walk of the axes of the intramolecular electric field potential (ligand field) to which a spin-bearing atomic center is exposed. The validity of the stochastic treatment presented here is not limited by the Redfield conditions. The general expression obtained for the time constant of electron spin relaxation in liquid phase reproduces the well-known result of Hubbard-Atkins-Kivelson theory if it is specialized to the case of systems with weak spin-orbit coupling.

\section{INTRODUCTION}

Since Kronig's ${ }^{1}$ and Van Vleck's ${ }^{2}$ pioneering work it is well recognized that, mediated through spin-orbit coupling (SOC), a random modulation of the interatomic electric field potential $V$ affects the spin and induces spin-lattice relaxation (SLR). The first application of the Kronig-Van Vleck mechanism, originally developed for crystals, to the description of spin relaxation in the liquid phase by Altshuler and Valiev $^{3}$ and, in more detail, by Kivelson ${ }^{4}$ was restricted to "stretching" modes of the collective motion, which modulate only the strength of $V$. The same idea has been exploited by McConnell in his "spin-orbit pulse" mechanism of SLR. ${ }^{5}$

Relaxation of rotational degrees of freedom could independently induce relaxation of a spin. This process has been described in terms of spin-rotational, 6,8 "rotational spin-orbit," 9,10 and "spin-orbit tunneling"" mechanisms of SLR. In its "pure" form SR relaxation operates in the gas phase where the change of molecular orientation may be described as a sequence of free rotations interrupted by collisions and the rotational (angular momentum) relaxation time $\tau_{N}$ is a physically well-defined parameter. Actually, this mechanism has been first introduced by Blombergen et al. ${ }^{12}$ in their studies of proton NMR in a gas. This "gas phase"type mechanism has been adapted to liquids and investigated in great details for molecules of different shape and for different models of molecular rotation in the condensed phase (see, e.g., Ref. 8 and references therein).

The theory of SR relaxation has been formulated in terms of the effective spin Hamiltonian: ${ }^{6,8}$

$$
H_{\text {sr }}=\mathbf{N} \hat{C} \mathbf{S}^{\text {eff }} \text {, }
$$

where $\mathbf{N}$ is the rotational angular momentum of the molecule, $\hat{C}$ is the spin-rotation coupling tensor, and $S^{\text {eff }}$ is the operator of effective spin. This interaction can lead to SLR, because both $\mathbf{N}$ and $\hat{C}$ are stochasticaly modulated by molecular collisions in a gas and by anisotropic intermolecular forces in a liquid. Usually it is assumed that the spin and rotational variables are weakly coupled. Consequently, the theory may be applied only in cases where the relaxation of rotational degrees of freedom proceeds at a much higher rate than the SLR process (Redfield limit). This automatically leads to an exponential decay of the initial spin orientation with the rate constant $1 / T_{s}$ which may be expressed in terms of the correlation function $K_{\mathrm{sr}}(t)$ of $H_{\mathrm{sr}}$, Eq. (1). In liquids the time scales of rotational (angular momentum) and orientational relaxation $\tau_{\theta l}$ are very different $\left(\tau_{N} \ll \tau_{\theta l}\right)$. Therefore, $K_{\mathrm{sr}}(t)$ can be factorized and $1 / T_{s}$ may be readily calculated (see, e.g. Ref. 8).

Nuclear spin is in general weakly coupled to the lattice. Therefore, the Redfield approximation is undoubtedly applicable for the description of nuclear SLR in the liquid phase (the case for which the theory was originally developed ${ }^{6}$ ). It should be clear from the above that in such a situation the following inequality holds true:

$$
T_{s} \gg \tau_{\theta l} \gg \tau_{N} \text {. }
$$

Atkins and Kivelson ${ }^{7}$ were the first to apply the spinrotational mechanism to the analysis of electron spin relaxation. Following Curl, ${ }^{13}$ they approximated the effective spin-rotational Hamiltonian [Eq. (1)] by the expression ${ }^{7,13}$

$$
H_{\mathrm{sr}} \approx-\mathbf{N}\left(\hat{I}^{-1} \delta \hat{g}\right) \mathbf{S}^{\text {eff. }} \text {. }
$$

Here $\delta \hat{g}=\hat{g}-g_{e} \hat{1}$, with $g_{e}$ the $g$ factor of the free electron, and $\hat{I}$ the molecular tensor of inertia. It should be stressed that this approximation is valid only if $\|\delta \hat{g}\| \ll 1$, which is a common case for organic radicals.

In Ref. 14 a geometrical approach to the problem was developed. It has been shown that in $S^{\text {eff }}=1 / 2$ species $S R$ interaction arises from a $U(2)$ non-Abelian gauge potential and can be described within the framework of the adiabatic rotation of effective spin (ARES) formalism (cf. Ref. 14, in the following referred to as ARES I) in terms of an effective spin-Hamiltonian:

$$
H_{\mathrm{ARES}}^{(L)}=-i A^{(L)}=-\boldsymbol{\omega} \delta \hat{\gamma} \mathbf{S}^{\mathrm{eff}} .
$$

Here $L$ denotes the lab frame, $A$ is the corresponding gauge potential, $\delta \hat{\gamma}=\hat{\gamma}-1$, and $\boldsymbol{\omega}=\mathbf{N} \hat{I}^{-1}$ is the angular velocity of molecular rotation. The tensor $\hat{\gamma}$ is defined by the expression $^{14,15}$

$$
\hat{\gamma} \mathbf{S}^{\text {eff }}=P_{\mathrm{KD}}(\mathbf{L}+\mathbf{S}) P_{\mathrm{KD}}
$$

Here $\mathbf{L}$ and $\mathbf{S}$ are the operators of electron orbital momentum and spin, $P_{\mathrm{KD}}$ is the projector onto the two-dimensional Hilbert space which is spanned by the ground Kramers doublet 
(KD). The specific form of $P_{\mathrm{KD}}$ and thus $\hat{\gamma}$, computed from Eq. (5), depends upon the eigenbasis of the Hamiltonian in the nonrotating system and reflects the degree of mixing between the eigenfunctions $\left|\alpha^{(L)}\right\rangle$ and $\left|\beta^{(L)}\right\rangle$ of the real spin operator $S_{Z_{L}}^{(L)}$. It has been pointed out ${ }^{14}$ that in the case of weak SOC $\delta \hat{\gamma} \approx \delta \hat{g}$, and thereby $H_{\mathrm{ARES}}^{(L)} \approx H_{\mathrm{sr}}^{(L)}$.

One should note that, independent of the particular model used to describe electron spin relaxation, the theoretically predicted upper limit of the SLR rate for $S^{\text {eff }}=\frac{1}{2}$-systems with weak coupling between spin and spatial degrees of freedom in magnetically dilute solutions is less than $10^{9} \mathrm{~s}^{-1}$ (see, e.g., Ref. 16 and references therein). However, for $\left(S^{\text {eff }}=\frac{1}{2}\right)$ paramagnetic complexes of high ligand symmetry (slightly distorted octahedral) extremely short values of electron SLR times $T_{s} \sim 10^{-11}-10^{-12} \mathrm{~s}$ have been reported. ${ }^{17} \mathrm{~A}$ relaxation time in the subnanosecond time scale has been estimated for hydroxyl radicals and some other organic and inorganic radicals of axial symmetry in liquid solutions. ${ }^{18,19}$ The basic common feature of these species is their partially unquenched orbital momentum and, resulting from it, a strong coupling between electron spin and spatial variables. Consequently, inequality (2) and Eq. (3) do not hold $\left(T_{s} \sim \tau_{\theta l}\right.$, or even shorter than $\left.\tau_{\theta l}\right)$ and the Redfield approximation is not valid. It appears, however, that so far there is no theoretical treatment of SR relaxation of electron spin in the condensed phase which is not based on the assumption of Redfield conditions.

In this paper it will be shown that ARES-type motion of the effective spin, which adiabatically follows the random walk of the main axes of the intramolecular electric (ligand) field tensor, is a suitable and most useful picture for describing spin-rotational relaxation. Applying the ARES approach we will provide a theoretical description of SR relaxation in $S^{\text {eff }}=1 / 2$ species beyond the Redfield limit, i.e., for situations with an arbitrary rate of stochastic modulation of the ARES Hamiltonian, Eq. (4). Our approach to the problem is valid for an arbitrary ratio between the basic "structural" parameters of a system: effective SOC constant $\lambda$ and the strength of its intramolecular electric (ligand) field potential $V$. In the limit of weak SOC the well-known expression for $1 / T_{s}$ that results from the Hubbard theory of SR relaxation in the liquid phase for systems with two orientational degrees of freedom $^{6-8}$ is reproduced.

\section{TIME EVOLUTION OF MAGNETIZATION}

Even in the absence of an external magnetic field the SLR process can be characterized by the decay of the projection of the ensemble averaged, $\langle\cdots\rangle$, electron spin on its original direction in space, which may be chosen as the axis $Z_{L}$ of the $L$ frame. If the lattice variables are "frozen" the evolution of the density operator $\rho^{(L)}(t)$ is governed by the Liouville equation $(\hbar=1)$

$$
\frac{d}{d t} \rho^{(L)}(t)=-i H_{L}^{\times}(0) \rho^{(L)}(t) .
$$

Following Refs. 14 and 20 we consider the Hamiltonian

$$
H_{L}(0)=\lambda \mathbf{L S}+V_{L}(0) \text {. }
$$

At $t=0$ the axes of the $L$ frame are oriented along the principal axes of the electric (ligand) field tensor $\hat{V}$. For atoms with an odd number of electrons in an environment of symmetry lower than cubic, it is possible that, among the eigenstates of $H_{L}(0)$ there are some exhibiting no other than Kramers degeneracy. ${ }^{21}$ Let us consider the situation in which such a Kramers doublet constitutes the ground term of the system. It is spanned by two kets: $\mid \overline{\left.\alpha^{(L)}\right\rangle}$ and $\mid \overline{\left.\beta^{(L)}\right\rangle}$.

Now let the molecule rotate. With no significant loss of generality we may take the origin of $L$ frame at the molecular center of mass and assume that the main axes of the molecular tensor of inertia coincide with the principal axes of the electric (ligand) field tensor. Since, in the case under consideration, $V, \lambda \gg k_{B} T$, any thermal molecular motion to which the real spin is coupled occurs at a much slower rate than the energy difference (in frequency units) between the lowest $\mathrm{KD}$ and higher eigenstates of $H_{L}$. In other words, the ground doublet is adiabatically isolated from the excited levels and, in order to calculate any observable quantity of a system, we only need the matrix elements of the corresponding operator within this lowest $\mathrm{KD}$. In such a case it is convenient to introduce the effective (formal) spin operator $\mathbf{S}^{\text {eff }}$ (cf. Ref. 21, and ARES I) acting in a two-dimensional Hilbert space in which an eigenbasis of $\left(S^{\text {eff }}\right)^{2}$ and $S_{Z_{L}}^{\text {eff(L) }}$ constitute the eigenvectors $\left|\overline{\alpha^{(L)}}\right\rangle$ and $\left|\overline{\beta^{(L)}}\right\rangle$. Molecular rotation results in the variation of the orientation of vector $S^{\text {eff }}(t)$ relative to its initial direction $\mathbf{S}^{\text {eff }}(0)$. Formally, this is performed by a linear transformation $U_{\mathrm{ARES}}(t)$ in the twodimensional Hilbert space of eigenfunctions of $S_{Z_{L}}^{\mathrm{eff}(L)} \cdot{ }^{14}$

In the rotating molecular frame $\left(M_{0}\right)$ the evolution of a reduced density operator of the ground $\mathrm{KD}$ $\left(\left|\overline{\alpha^{(M)}}\right\rangle,\left|\overline{\beta^{(M)}}\right\rangle\right)$ :

$$
\tilde{\rho}^{(M)}(t)=\hat{U}_{\mathrm{ARES}}^{(M)}(t) \tilde{\rho}^{(M)}(0), \quad \tilde{\rho}^{(M)}(0)=\tilde{\rho}^{(L)}(0)
$$

is governed by the propagator $\hat{U}_{\mathrm{ARES}}^{(M)}(t)$ which is defined in the $(2 \times 2)^{2}$ Liouville space

$$
\hat{U}_{\mathrm{ARES}}^{(M)}(t)=T \exp \left\{-i \int_{0}^{t} H_{\mathrm{ARES}}^{(M) \times}\left(t^{\prime}\right) d t^{\prime}\right\} ;
$$

where $T$ is the time-ordering operator. ${ }^{14}$

Consider now the time behavior of the following quantity:

$$
M_{Z_{L}}^{\text {eff }}(t)=\operatorname{Tr}\left\langle S_{Z_{L}}^{\text {eff(M) }} \tilde{\rho}^{(M)}(t)\right\rangle .
$$

The transformation which relates the irreducible tensor operators in the lab frame to those in the moving molecular frame is

$$
S_{q_{L}}^{\mathrm{eff}(M)}=\hat{\hat{R}}\left(\Omega_{L M}\right) S_{q_{L}}^{\mathrm{eff}(L)}=\sum_{q_{L}^{\prime}} R_{q_{L}^{\prime}, q_{L}}^{1}\left(\Omega_{L M}\right) S_{q_{L}^{\prime}}^{\mathrm{eff}(L)} .
$$

Here $q_{L}=0_{L} \equiv Z_{L}, \Omega_{L M}=\left\{\alpha_{L M}, \beta_{L M}, \chi_{L M}\right\}$ is the set of Euler angles defining the instantaneous orientation of the principal axes of the electric (ligand) field tensor $\hat{V}$ ( $M$ frame) relative to the $L$ frame; $\hat{R}$ is the Liouville space operator of finite rotation relating two systems of coordinates, the $R_{q_{L}^{\prime}, q_{L}}^{1}$ are the Wigner rotation matrices. $\hat{R}\left(\Omega_{L M}\right)$ is given by 


$$
\begin{aligned}
\hat{R}\left(\Omega_{L M}\right)= & \exp \left[i \alpha_{L M}\left(L_{Z_{M}}^{\times}+S_{Z_{M}^{-}}^{\times}\right)\right] \exp \left[i \beta_{L M}\left(L_{Y_{M}}^{\times}+S_{Y_{M}}^{\times}\right)\right] \\
& \times \exp \left[i \chi_{L M}\left(L_{Z_{M}}^{\times}+S_{Z_{M}}^{\times}\right)\right] .
\end{aligned}
$$

Substituting Eqs. (8) and (11) into Eq. (10), we obtain

$$
M_{Z_{L}}^{\text {eff }}(t)=\operatorname{Tr} \sum_{q_{L}} S_{q_{L}}^{\mathrm{eff}(L)}\left\langle R_{q_{L}, 0}^{1}\left[\Omega_{L M}(t)\right] \hat{U}_{\mathrm{ARES}}^{(M)}(t)\right\rangle \tilde{\rho}^{(L)}(0)
$$

The random character of the molecular rotation in a liquid leads to a stochastic modulation of $H_{\mathrm{ARES}}^{(M)}$. Thus it is necessary to average Eq. (13) over all realizations of the random process $H_{\mathrm{ARES}}^{(M)}(t)$ or, in other words, to find the relaxation operator

$$
\hat{\Phi}_{q_{L}, 0_{L}}(t)=\left\langle R_{q_{L}, 0_{L}}^{1}\left[\Omega_{L M}(t)\right] \hat{U}_{\mathrm{ARES}}^{(M)}(t)\right\rangle .
$$

Knowing $\hat{\Phi}_{q_{L}, 0_{L}}(t)$ it is straightforward to calculate the decay of the observable $M_{Z_{L}}^{\text {eff }}(t)$.

\section{KINETIC EQUATION}

It is well-known that in the condensed phase the angular velocity (angular momentum) of a molecule relaxes on a much shorter time scale, $\tau_{N}$, than the orientational coordinates. Thus for times $t \gg \tau_{N}$, it is legitimate to eliminate the fast variables and to assume that the process of random variation of the molecular orientation is a stationary Markovian process. ${ }^{8,22}$ In such a case the conditional probability $P\left(\Omega_{L M}, \Omega_{L M}^{U} ; t-t_{0}\right)$ of finding the orientation of the molecular framework equal to $\Omega_{L M}$ at the end of the time interval $\left(t_{0}, t\right)$, if it is known to have been equal to $\Omega_{L M}^{0}$ at its beginning $\left(t_{0}=0\right)$ satisfies the Kolmogorov-Feller equation ${ }^{23}$

$$
\begin{aligned}
& \frac{\partial}{\partial t} P\left(\Omega_{L M}, \Omega_{L M}^{0} ; t\right) \\
& =-\tau_{c}^{-1}\left[P\left(\Omega_{L M}, \Omega_{L M}^{0} ; t\right)-\int P\left(\Omega_{L M}^{\prime}, \Omega_{L M}^{0} ; t\right)\right. \\
& \left.\quad \times f\left(\Omega_{L M}^{\prime}, \Omega_{L M}\right) d \Omega_{L M}^{\prime}\right], \\
& P\left(\Omega_{L M}, \Omega_{L M}^{0} ; 0\right)=\delta\left(\Omega_{L M}-\Omega_{L M}^{0}\right) .
\end{aligned}
$$

Here $\tau_{c}$ is a mean time between the variation of the molecular orientation. The function $f\left(\Omega_{L M}^{\prime}, \Omega_{L M}\right)$ describes the probability density for jumping from $\Omega_{L M}^{\prime}$ to $\Omega_{L M}$ in one jump. Since the problem is formulated in the $M$ frame, the sets of Euler angles $\Omega_{L M}^{\prime}, \Omega_{L M}$, and $\Omega_{L M}^{0}$ are chosen to define the orientation of the rotating molecular framework relative to the immobile $L$ frame, therefore $\Omega_{L M}^{0}$ in Eqs. (15) and (16) equals zero.

If, e.g., molecular rotation is accomplished by small (i.e., much less than $2 \pi$ ) steps in the angular space it may be considered as a Debye rotational diffusion ${ }^{8,22}$ at times $t \gg \tau_{N}$. The solution of Eq. (15) for the case of continuous diffusion in the angular space is well-known ${ }^{24}$ and in principle could be used in Eq. (17) to find an average over all realizations of $\Omega_{L M}(t)$ :

$$
\begin{aligned}
M_{Z_{L}}^{\mathrm{eff}}(t)= & \operatorname{Tr} \sum_{q_{L}} S_{q_{L}}^{\mathrm{eff}(L)}\left\{\int d \Omega_{L M} P\left(\Omega_{L M}, 0 ; t\right)\right. \\
& \left.\times \hat{\Phi}_{q_{L}, 0_{L}}\left(\Omega_{L M}, t\right) \tilde{\rho}^{(L)}(0)\right\} .
\end{aligned}
$$

Here the partially averaged relaxation operator $\hat{\Phi}_{q_{L}, 0_{L}}\left(\Omega_{L M}, t\right.$ ) (averaged only over those realizations of diffusional trajectories which have identical values $\Omega_{L M}$ at the instant $t$ ) has been introduced, whereby

$\hat{\Phi}_{q_{L}, 0_{L}}(t)=\int d \Omega_{L M} P\left(\Omega_{L M}, 0 ; t\right) \hat{\Phi}_{q_{L}, 0_{L}}\left(\Omega_{L M}, t\right)$.

It is convenient to describe the decay of $M_{Z_{L}}^{\text {eff }}(t)$ in terms of the averaged operator

$$
\sigma_{q_{L}}(t) \equiv \int d \Omega_{L M} P\left(\Omega_{L M}, 0 ; t\right) \hat{\Phi}_{q_{L}, 0_{L}}\left(\Omega_{L M}, t\right) \tilde{\rho}^{(L)}(0)
$$

by use of which Eq. (17) takes the form

$$
M_{Z_{L}}^{\mathrm{eff}}(t)=\operatorname{Tr} \sum_{q_{L}} S_{q_{L}}^{\mathrm{eff}(L)}\left\{\sigma_{q_{L}}(t)\right\} .
$$

Let us multiply Eq. (15) by $\hat{\Phi}_{q_{L}, 0_{L}}\left(\Omega_{L M}, t\right) \tilde{\rho}^{(L)}(0)$ and integrate the result over $\Omega_{L M}$, like in Eq. (17). This procedure gives

$$
\begin{aligned}
\frac{\partial}{\partial t} \sigma_{q_{L}}(t)= & -\tau_{c}^{-1}\left[\sigma_{q_{L}}(t)-\int \hat{\hat{\Phi}}_{q_{L}, 0_{L}}\left(\Omega_{L M}, t\right) \tilde{\rho}^{(L)}(0)\right. \\
& \left.\times d \Omega_{L M} \int P\left(\Omega_{L M}^{\prime}, 0 ; t\right) f\left(\Omega_{L M}^{\prime}, \Omega_{L M}\right) d \Omega_{L M}^{\prime}\right]
\end{aligned}
$$

To proceed further we must specify the partially averaged relaxation operator $\Phi_{q_{L}, 0_{L}}\left(\Omega_{L M}, t\right)$ explicitly. In what follows the approximate form of $\hat{\Phi}_{q_{L}, 0_{L}}\left(\Omega_{L M}, t\right)$ will be used, which is exact in the limit of short times wherein only infinitesimally small angles of molecular (ligand field) reoricntation may be rcached (see also Ref. 15):

$$
\hat{\Phi}_{q_{L}, 0_{L}}\left(\Omega_{L M}, t\right)=R_{q_{L}, 0_{L}}^{1}\left(\Omega_{L M}\right) \hat{\hat{R}}^{\mathrm{eff}}\left(\Omega_{L M}\right)
$$

Here

$$
\begin{aligned}
& \hat{R}^{\mathrm{eff}}\left[\Omega_{L M}\right]= \exp \left(i \gamma_{\|} \alpha_{L M} S_{Z_{M}}^{\mathrm{effx}}\right) \exp \left(i \gamma_{\perp} \beta_{L M} S_{Y_{M}}^{\mathrm{effx}}\right) \\
& \times \exp \left(i \gamma_{\|} \chi_{L M} S_{Z_{M}}^{\text {effx }}\right), \\
& \gamma_{\|} \equiv \hat{\gamma}_{Z_{M}, Z_{M}} ; \quad \gamma_{\perp} \equiv \hat{\gamma}_{X_{M}, X_{M}}=\hat{\gamma}_{Y_{M}, Y_{M}} .
\end{aligned}
$$

Note that in an isotropic medium $f\left(\Omega_{L M}^{\prime}, \Omega_{L M}\right)$ $=f\left(\Omega_{L M}^{\prime}-\Omega_{L M)} \equiv f(\tilde{\Omega})\right.$. That allows us, using the relationships $\hat{R}(\tilde{\Omega}) \cong \hat{R}(\Omega) \hat{\hat{R}}\left(-\Omega^{\prime}\right)$ and $d \tilde{\Omega}=d \Omega$, to represent the integral term of Eq. (21) (see also Ref. 25) as 


$$
\begin{gathered}
\sum_{q_{1 L}} \int R_{q_{L}, q_{1 L}}^{1}(\tilde{\Omega}) \hat{R}^{\mathrm{eff}}(\tilde{\Omega}) f(\tilde{\Omega}) d \tilde{\Omega}\left\{\int P\left(\Omega_{L M}^{\prime}, 0 ; t\right)\right. \\
\left.\quad \times R_{q_{1 L}, 0_{L}}^{1}\left(\Omega_{L M}^{\prime}\right) \hat{R}^{\mathrm{eff}}\left(\Omega_{L M}^{\prime}\right) \tilde{\rho}^{(L)}(0) d \Omega_{L M}^{\prime}\right\} \\
=\sum_{q_{1 L}} \int R_{q_{L}, q_{1 L}}^{1}(\tilde{\Omega}) \hat{R}^{\hat{e f f}}(\tilde{\Omega}) \sigma_{q_{1 L}}(t) f(\tilde{\Omega}) d \tilde{\Omega} .
\end{gathered}
$$

Thus Eq. (21) is closed relative to $\sigma_{q_{L}}(t)$ and has the form

$$
\frac{\partial}{\partial t} \sigma_{q_{L}}(t)=-\sum_{q_{1 L}}{\stackrel{\hat{T}}{\hat{T}_{q_{L}}, q_{1 L}}}_{q_{q_{1 L}}} \sigma_{q}(t)
$$

where

$$
\begin{aligned}
\hat{T}_{q_{L}, q_{1 L}}^{\mathrm{fff}} \sigma_{q_{1 L}}(t)= & \tau_{c}^{-1} \int[\delta(\tilde{\Omega})-f(\tilde{\Omega})] \\
& \times \hat{\Phi}_{q_{L}, q_{1_{L}}}(\tilde{\Omega}) \sigma_{q_{1 L}}(t) d \tilde{\Omega} .
\end{aligned}
$$

The initial condition for Eq. (25) is obtained from the definitions of $\sigma_{q_{L}}(t)$ [Eq. (19)], and $\hat{R}^{\text {eff }}(\Omega)$ [Eq. (23)]:

$$
\sigma_{q_{L}}(0)=\delta_{q_{L}, 0_{L}} \tilde{\rho}^{(L)}(0)
$$

The kinetic equation (25) constitutes the basis for the description of the SLR process in the ground $\mathrm{KD}$ for systems with an arbitrarily strong SOC. Formally, this equation looks like the homogeneous differential equations of the impact theory ${ }^{22}$ with the "collisional operator" [Eq. (26)]. The basic difference is the absence of a Hamiltonian which governs the dynamical evolution of the system on the "free path" between "collisions," i.e., jumps of the molecular framework in the angular space. The Hamiltonian is hidden in the definition of the $\hat{R}^{\text {eff }}[\mathrm{Eq}$. (23)] from which it is readily seen that the following equality holds true:

$$
\hat{R}^{\mathrm{eff}}(\Omega)=\hat{R}^{(1 / 2)}\left(\Omega^{\mathrm{eff}}\right),
$$

where $\Omega^{\text {eff }}=\left\{\gamma_{\|} \alpha, \gamma_{\perp} \beta, \gamma_{\|} \chi\right\}$. Thus, as expected, the response of the effective spin on the variation of the molecular orientation depends upon the strength of the coupling between the spatial and spin degrees of freedom, which is reffected in the parameters $\gamma_{\|}$and $\gamma_{\perp}$.

\section{RELAXATION TIME}

Consider an atom with a single electron $(\lambda>0)$ or hole $(\lambda<0)$ in the $p$-valence shell and exposed to an axially symmetric environment. The action of $V_{L}$ [Eq. (7)] in the space of $p$ functions may be expressed in terms of the $T_{2 n}$ component of the set of unit irreducible tensor operators of rank- $2,{ }^{14,20}$

$$
V_{L}(0)=\sqrt{\frac{2}{3}} \Delta T_{20}^{(L)},
$$

where $\Delta=-(3 / 2) \hat{V}_{Z_{L}, Z_{L}}$ defines the splitting of $p$ orbitals in the absence of SOC under the action of the interatomic electric field potential; $\hat{V}_{Z_{L}, Z_{L}}$ is the corresponding main value of the electric field tensor, and $\hat{V}_{X_{L}, X_{L}}=\hat{V}_{Y_{L}, Y_{L}}$
$=\Delta / 3$ for axial symmetry. In this simple model case we may readily find the eigenfunctions of $H_{L}$ [Eq. (7)] and calculate the basic parameters of the theory ${ }^{14}$

$$
\gamma_{\|}=1 ; \quad \gamma_{\perp}=\cos ^{2} x+2 \sqrt{2} \sin x \cos x .
$$

The degree of mixing between the different spin-orbital states is expressed by $x$ and is determined by the ratio of the electric field splitting parameter $\Delta$ and the effective SOC constant $\lambda$ :

$$
\tan (x)=\frac{\sqrt{2} \lambda}{\lambda / 2-\Delta-\sqrt{(\lambda / 2-\Delta)^{2}+2 \lambda^{2}}}
$$

with $-(\pi / 2) \leqslant x \leqslant(\pi / 2)$.

To proceed further, let us multiply Eq. (25) by $S_{q_{L}}^{\text {eff( }(L)}$ and take the trace from both sides. As is shown in the Appendix, in the case of linear molecules this procedure leads to the following equation:

$$
\frac{\partial}{\partial t} M_{q_{L}}^{\mathrm{eff}}(t)=-\sum_{q_{1 L}} A_{q_{L}, q_{1 L}} M_{q_{1 L}}^{\mathrm{eff}}(t) .
$$

Here

$$
\begin{aligned}
M_{q_{L}}^{\mathrm{eff}}(t) \equiv & \operatorname{Tr} \sigma_{q_{L}}(t) S_{q_{L}}^{\mathrm{eff}(L)} \\
A_{q_{L}, q_{1 L}} \equiv & \tau_{c}^{-1} \int_{0}^{1} d(\cos \tilde{\beta})[\delta(\cos \tilde{\beta}-1) \\
& -f(\cos \tilde{\beta})] R_{q_{L}, q_{1 L}}^{1}(\tilde{\beta}) R_{q_{L}, q_{1 L}}^{1}\left(\gamma_{\perp} \tilde{\beta}\right) .
\end{aligned}
$$

Since $M_{Z_{L}}^{\text {eff }}(t)=\Sigma_{q_{L}} M_{q_{L}}^{\text {eff }}(t)$ [Eq. (19)], the spin relaxation time, defined as

$$
T_{s} \equiv \int_{0}^{\infty} d t M_{Z_{L}}^{\mathrm{eff}}(t) / M_{Z_{L}}^{\mathrm{eff}}(0)
$$

can be found by Laplace transformation of Eq. (32):

$$
T_{s}=\sum_{q_{L}, q_{1 L}}\left\{A^{-1}\right\}_{q_{L}, q_{1 L}} M_{q_{1 L}}^{\mathrm{eff}}(0) / M_{Z_{L}}^{\mathrm{eff}}(0)
$$

The initial condition for Eq. (32) is obtained from Eq. (27):

$$
M_{q_{L}}^{\text {eff }}(0)=\delta_{q_{L}, 0} M_{Z_{L}}^{\text {eff }}(0) .
$$

Thus the inversion of $3 \times 3$ matrix $A_{q_{L}, q_{1 L}}$ gives

$$
\frac{1}{T_{s}}=\frac{a^{2}-b^{2}}{a-2 b} \tau_{c}^{-1}
$$

where

$$
\begin{aligned}
a= & \int_{0}^{1} d(\cos \tilde{\beta})[\delta(\cos \tilde{\tilde{\beta}}-1)-f(\cos \tilde{\beta})] \\
& \times\left[\cos \left(1+\gamma_{\perp}\right) \tilde{\beta}+\cos \left(1-\gamma_{\perp}\right) \tilde{\beta}\right] / 2, \\
b= & \int_{0}^{1} d(\cos \tilde{\beta})[\delta(\cos \tilde{\beta}-1)-f(\cos \tilde{\beta})] \\
& \times\left[\cos \left(1-\gamma_{\perp}\right) \tilde{\beta}-\cos \left(1+\gamma_{\perp}\right) \tilde{\beta}\right] / 2 .
\end{aligned}
$$

It is easy to see from Eqs. (30) and (31) that if there is no connection between the real spin $\mathbf{S}$ and orbital momentum $\mathbf{L}$ 
$(\lambda=0)$ the degenerate functions of the corresponding $\mathrm{KD}$ span a complete representation of the spinor group $S U(2)$. In this limit $\left|\bar{\alpha}^{\bar{L}}\right\rangle=\left|\alpha^{L}\right\rangle,\left|\widetilde{\beta}^{L}\right\rangle=\left|\beta^{L}\right\rangle, \hat{\gamma}=1$, and Eq. (38) gives $1 / T_{s}=0$. Due to SOC the spin and orbital variables are coupled. At the same time, in the presense of the anisotropic electric field potential the orbital momentum is no longer a constant of motion $\left(L^{2}\right.$ does not commute with $V$ ). As a result, if $V, \lambda \neq 0$, the eigenstates of $H_{L}$ [Eq. (7)] span neither a representation of $S U(2)$ nor a $S U(2) \times S O(3)$ double group. If $\Delta \gg \lambda$, the spin and spatial degrees of freedom are weakly coupled [Hund case (b)]. In this limit $\left|1-\gamma_{\perp}\right| \ll 1$ (see also Ref. 14). As a result, Eqs. (38)-(40) yield

$$
1 / T_{s} \cong \frac{4}{3}\left(1-\gamma_{\perp}\right)^{2} D_{R}
$$

where

$$
D_{R}=\int_{0}^{1} d(\cos \tilde{\beta}) f(\cos \tilde{\beta}) \tilde{\beta}^{2} / 4 \tau_{c}
$$

is the coefficient of rotational diffusion for a linear rotator (systems with two rotational degrees of freedom). ${ }^{24}$ This result is independent of the particular form of $f(\cos \tilde{\beta})$, as it should be within the region of applicability of stochastic perturbation theory.

It has been shown in Ref. 14 that if $\lambda /\|V\| \ll 1$, in the first nonzero order of perturbation theory,

$$
\left(1-\gamma_{\perp}\right)^{2}=\left(g_{e}-g_{\perp}\right)^{2} \text {. }
$$

Here $g_{\perp}$ is the $\hat{g}_{Y_{L}, X_{L}}=\hat{g}_{X_{L}, X_{L}}$ main value of the $g$ tensor. Thus Eq. (41) reproduces the well-known result of Hubbard's theory (see, e.g., Ref. 8 and references therein). Note, however, that in our derivation of Eq. (41) we did not treat the problem in terms of an effective Hamiltonian of spinrotational interaction, Eq. (2), and thus avoided using the Hubbard relation between $\tau_{N}$ and the orientational relaxation time $\tau_{\theta l}$ of rank $l$ tensors. ${ }^{6,8,22}$

The case of our primary interest is $\|V\| \geqslant \lambda \gg k_{B} T$ [Hund case (a)], a situation encountered in paramagnetic complexes of high symmetry, axially symmetric and atomic radicals with heavy atoms. In this case the spin and spatial degrees of freedom are strongly coupled, $\gamma_{\perp} \ll 1$, and the effective spin closely follows the orientation of the molecular framework. ${ }^{14}$ It is readily seen that if, e.g., $\Delta=9 \lambda / 4$, Eqs. (30) and (31) give $\gamma_{\perp}=0$, and from Eqs. (38) $-(40)$ we immediately obtain

$$
\begin{aligned}
T_{s}^{-1} & =\tau_{c}^{-1} \int_{0}^{1} d(\cos \tilde{\beta})[\delta(\cos \tilde{\beta}-1)-f(\cos \tilde{\beta})] \cos \tilde{\beta} \\
& =\tau_{\theta 1}^{-1} .
\end{aligned}
$$

Consequently, in the case of relatively strong SOC $\left(\gamma_{\perp} \ll 1\right)$ the SLR time for linear molecules should be close to the orientational relaxation time $\tau_{\theta 1}$.

Note that in the model of continuous diffusion $f(\cos \tilde{\beta})$ is different from zero only for small $\tilde{\beta}$. Thereby we may expand $\cos \left(1 \pm \gamma_{\perp}\right) \tilde{\beta}$ in Eqs. (39) and (40) in powers of $\tilde{\beta}$. Dropping terms of higher than the second order in $\tilde{\beta}$ we obtain the following result ${ }^{26}$ :

$$
T_{s}^{-1}=\frac{\left(1-\gamma_{\perp}\right)^{2}\left(1+\gamma_{\perp}\right)^{2}}{\left(1+\gamma_{\perp}\right)^{2}+2 \gamma_{\perp}}\left(2 D_{R}\right)
$$

$$
0 \leqslant \gamma_{\perp} \leqslant 1
$$

It is easy to verify that in the respective limiting cases this expression reproduces Eqs. (41) and (44) (note that for linear rotators in the diffusion approximation $\tau_{\theta \mathrm{l}}^{-1}=2 D_{R}{ }^{24}$ ).

\section{DISCUSSION}

Traditionally the process of spin-lattice relaxation is described as a result of nonadiabatic (instantaneous) stochastic modulation of the dynamical (regular) motion of spin. The random character of the perturbation results from thermal fluctuations of spatial variables which are usually modeled by Markovian or Gaussian-type stochastic process (see, e.g., Refs. $8,16,21$, and 22 , and references therein). This nonadiabatic kinetic theory cannot describe the evolution of the system over times comparable to or less than the "switching" time $\tau_{v}$ of the perturbation (the time interval in which the variation of the Hamiltonian takes place). The nonadiabatic approach to spin relaxation is well justified when the energy separation between the eigenstates of the Hamiltonian coupled by the time-dependent perturbation is much smaller than $\tau_{0}^{-1} \sim 10^{11}-10^{13} \mathrm{~s}^{-1}\left(\ll k_{B} T\right)$. For $S=1 / 2$ systems this is obviously the case when the frequency of internal motions is determined by Zeeman interactions. The nonadiabatic theory of SLR may still be appropriate for triplet molecules and paramagnetic complexes with $S^{\text {eff }} \geqslant 1$, where the zero-field splitting is usually lower than $10 \mathrm{~cm}^{-1}$.

The situation is different, however, if the energy gap between the terms coupled by the perturbation is determined by the electronic Hamiltonian $\left(\left\|H^{\times}\right\| \geqslant 10^{3} \mathrm{~cm}^{-1}\right)$, i.e., becomes much larger than the inverse correlation time of any thermal molecular motion. In this case the random perturbation of a system has a qualitatively different, namely an adiabatic, character.

Free radicals and paramagnetic complexes with $S^{\text {eff }}=1 / 2$ represent an important class of systems in which the spinorbit mixed Kramers doublet constitutes the adiabatically isolated degenerate electronic ground state. Actually, the energy difference between the orbitally nondegenerate ground state and the lowest excited electronic term coupled by SOC to the ground one is of the order of the intramolecular (ligand) electric field potential, which is usually much larger than $k_{B} T$. As a result, in these species the lowest $\mathrm{KD}$ is adiabatically isolated from the higher eigenstates of the molecular Hamiltonian.

In Ref. 14 the effect of regular molecular rotations, as far as they represent an adiabatic perturbation to the electronic system, has been adequately described as an adiabatic rotation of effective spin. It has been shown that the action of molecular rotation on the effective spin docs not depend on the angular velocity at which the molecule passes a given rotational trajectory, but only on the geometry of that trajectory, i.e., it can be described in purely geometric terms. Furthermore, the analysis that has been made in that work clearly shows that only in the case of weak SOC, the traditional representation of the effective spin-Hamiltonian of spin-rotational interaction, is equivalent to the more generally valid ARES Hamiltonian. 
In the present paper the theory of spin-rotational relaxation in systems with $S^{\text {eff }}=1 / 2$ has been developed within the framework of the ARES approach. It has been shown that spin-rotational relaxation results from the rotational fluctuations of the anisotropic intramolecular electric field potential (ligand field) to which the spin bearing atom is exposed. When this field is strong enough $\left(V \gg k_{B} T\right)$ the effective spin of the system follows the time variation of the $M$ frame adiabatically. In the $M$ frame, the ARES-type motion of the effective spin appears as if it were driven by a "fictitious" internal magnetic field, $\mathbf{f}=-\boldsymbol{\omega} \hat{\gamma}$. Correspondingly, the random walk of the principal axes of the tensor $\hat{V}$ in the angular space induces the SR relaxation.

Thus we come to the important qualitative conclusion: as long as the time variation of $\hat{V}$ in the angular space represents an adiabatic perturbation to the electronic system the spin-rotational relaxation in $S^{\text {eff }}=1 / 2$ species is a result of stochastic modulation of the molecular orientation, irrespective of the values of angular velocity at which the molecule passes the random trajectory in the angular space. Equivalently, in purely geometric terms, the ARES mechanism of spin-rotational relaxation originates from the action of a fluctuating Wilczek-Zee gauge potential (see Refs. 14, and references therein). Consequently, this process, traditionally attributed to the gas phase or nonviscous liquids, should be considered as an important one for most $S^{\text {eff }}=1 / 2$ systems even with only partially "unfrozen" rotational degrees of freedom, e.g., in hydrogen-bonded liquids, polymer solutions, and in the solid phase.

For the sake of clarity it seems of interest to compare the ARES mechanism of the spin-rotational relaxation with the "rotational spin-orbit" model of SLR. ${ }^{9,10}$ In the latter, spin relaxation is described as a response of $\mathbf{S}$ to the fluctuations of $\mathbf{L}$ in the angular space. Formally, the spin-orbit Hamiltonian $\lambda \mathbf{L S}$ was reduced to a time-dependent effective spin Hamiltonian by evaluating the matrix elements of $L$ in the molecular frame, with the implicit assumption that the orbitals do rotate adiabatically with the $M$ frame, whereas the effective spin does not. ${ }^{9,10}$ In its effect to the spin, rotation of the orbits was considered as a Gaussian-type ${ }^{9}$ or Markovian ${ }^{10}$ stochastic process. It should be clear from the above, that this approach to the problem is not valid when the energy gap between the ground Kramers doublet and the lowest excited electronic state spin-orbitally coupled to the ground one is more than $k_{B} T$. In this case one should consider the adiabatic motion of the full $(L, S)$ system in the rotating intramolecular electric field before the calculation of any macroscopical observable (see also Ref. 20, where the limit $V \ll k_{B} T$ has been considered in some detail).

In this paper the principles of treating spin-rotational relaxation in $S^{\text {eff }}=1 / 2$ systems by the ARES mechanism have been demonstrated. An atom with a single electron or hole in the $p$ shell belonging to a linear molecule rotating in an isotropic liquid, represents the simplest model case that allows us to come to first practical results. However, the general formulation of the problem in terms of the ARES kinetic equation (25) allows one to treat the action of the rotationally modulated electric (ligand) field potential on the spin bearing atom (also with $d$ and $f$ electrons) within many different models of thermal motion and molecular shape. An application to the problem of electron angular momentum relaxation in liquid solutions of highly symmetric paramagnetic complexes with $S^{\text {eff }}=1 / 2$ is now in progress.

\section{ACKNOWLEDGMENTS}

Financial support of this work by the Deutsche Forschungsgemeinschaft and the grant of an Alexander von Humboldt fellowship to Y. A. S. is gratefully acknowledged.

\section{APPENDIX}

Multiplying Eq. (25) by $S_{q_{L}}^{\operatorname{eff}(L)}$ and taking the trace we obtain

$$
\begin{aligned}
\frac{\partial}{\partial t} \operatorname{Tr}\left\{S_{q_{L}}^{\mathrm{eff}(L)} \sigma_{q_{L}}(t)\right\} \\
=-\tau_{c}^{-1} \sum_{q_{1 L}} \int[\delta(\tilde{\Omega})-f(\tilde{\Omega})] R_{q_{L}, q_{1 L}}^{1}(\tilde{\Omega}) \\
\quad \times \operatorname{Tr}\left\{S_{q_{L}}^{\mathrm{eff}(L)} \hat{R}^{(1 / 2)}\left(\tilde{\Omega}^{\mathrm{eff}}\right) \sigma_{q_{1 L}}(t)\right\} d \tilde{\Omega}
\end{aligned}
$$

Since

$$
\begin{aligned}
& \operatorname{Tr}\left\{S_{q_{L}}^{\mathrm{eff}(L)} \hat{R}^{1 / 2}\left(\tilde{\Omega}^{\mathrm{eff}}\right) \sigma_{q_{1 L}}(t)\right\} \\
& \quad=\operatorname{Tr}\left\{\sigma_{q_{1 L}}(t) \hat{R}^{(1 / 2)}\left(-\tilde{\Omega}^{\mathrm{eff}}\right) S_{q_{L}}^{\mathrm{eff}(L)}\right\}
\end{aligned}
$$

and

$$
\hat{R}^{(1 / 2)}\left(-\tilde{\Omega}^{\mathrm{eff}}\right) S_{q_{L}}^{\mathrm{eff}(L)}=\sum_{Q} R_{Q, q_{L}}^{1}\left(-\tilde{\Omega}^{\mathrm{eff}}\right) S_{Q}^{\mathrm{eff}(L)},
$$

Eq. (A1) takes the form

$$
\begin{aligned}
\frac{\partial}{\partial t} \operatorname{Tr}\left\{S_{q_{L}}^{\mathrm{eff}(L)} \sigma_{q_{L}}(t)\right\} \\
=-\tau_{c}^{-1} \sum_{Q, q_{1 L}} \int[\delta(\tilde{\Omega})-f(\tilde{\Omega})] R_{q_{L}, q_{1 L}}^{1}(\tilde{\Omega}) \\
\quad \times R_{Q, q_{L}}^{1}\left(-\tilde{\Omega}^{\mathrm{eff}}\right) \operatorname{Tr}\left\{\sigma_{q_{1 L}}(t) S_{Q}^{\mathrm{eff}(L)}\right\} d \tilde{\Omega} .
\end{aligned}
$$

For linear molecules in the isotropic liquid the kernel

$$
f(\tilde{\Omega})=f(\cos \tilde{\beta}) / 4 \pi^{2} .
$$

Thus, substituting Eq. (A3) into Eq. (A2) and using the exact expressions for Wigner rotation matrices, ${ }^{27}$ after integration over $\{\tilde{\alpha}, \tilde{\chi}\}$ and summation over $Q$, we obtain the closed relative to $M_{q_{L}}(t)$, Eq. (32).

${ }^{1}$ R. de L. Kronig, Physica 6, 33 (1939).

${ }^{2}$ J. Van Vleck, Phys. Rev. 57, 426 (1940).

${ }^{3}$ S. A. Altshuler and K. A. Valiev, Sov Phys. JETP 8, 661 (1959).

${ }^{4}$ D. Kivelson, J. Chem. Phys. 45, 1324 (1966).

${ }^{5}$ H. McConnell, J. Chem. Phys. 34, 13 (1961).

${ }^{6}$ P. S. Hubbard, Phys. Rev. 131, 1155 (1963).

${ }^{7}$ P. W. Atkins and D. Kivelson, J. Chem. Phys. 44, 169 (1966).

${ }^{8} \mathrm{~J}$. McConnell, The Theory of Nuclear Magnetic Relaxation in Liquids (Cambridge University, Cambridge, 1987).

${ }^{9}$ D. Kivelson and G. Collins, Paramagnetic Resonance, Proc. Intern. First Conf. Jerusalem (1962), Vol. 2, p. 496.

${ }^{10} \mathrm{~J}$. H. Freed, J. Chem. Phys. 49, 376 (1968). 
${ }^{11}$ J. H. Freed and R. G. Kooser, J. Chem. Phys. 49, 4715 (1968); R. G. Kooser, W. V. Volland, and J. H. Freed, ibid. 50, 5243 (1969); M. R. Das, S. B. Wagner, and J. H. Freed, ibid. 52, 5404 (1970).

${ }^{12}$ N. Bloembergen, E. M. Parcell, and R. V. Pound, Phys. Rev. 73, 679 (1948).

${ }^{13}$ R. F. Curl, Ir., J. Chem. Phys. 37, 779 (1962); Mol. Phys. 9, 585 (1965).

${ }^{14}$ U. E. Steiner and Yu. A. Serebrennikov, J. Chem. Phys. 100, 7503 (1994).

${ }^{15}$ U. E. Steiner and D. Bür $\beta$ ner, Z. Phys. Chem. N. F. 169, 159 (1990).

${ }^{16}$ I. V. Khudyakov, Yu. A. Serebrennikov, and N. J. Turro, Chem. Rev. 93, 537 (1993).

${ }^{17}$ L. Banci, I. Bertini, and C. Luchinat, Nuclear and Electron Relaxation (VCH, Weinheim, 1991); D. M. Doddrell, D. T. Pegg, M. R. Bendall, and A. K. Gregson, Aust. J. Chem. 30, 1635 (1977); D. Bür ßner, H.-J. Wolff, and U. E. Steiner, Z. Phys. Chem. N.F. (in press).

${ }^{18}$ B. Brocklehurst, J. Chem. Soc., Faraday Trans. 2 75, 123 (1979).

${ }^{19}$ C. Bohne, M. S. Alnajjar, D. Griller, and C. Scaiano, J. Am. Chem. Soc. 113, 1444 (1991).

${ }^{20}$ Yu. A. Serebrennikov and U. E. Steiner, Z. Phys. Chem. N.F. (in press).
${ }^{21}$ A. Abragam and B. Bleany, Electron Paramagnetic Resonance of Transition Ions in Crystals (Academic, New York, 1970).

${ }^{22}$ A. I. Burshtein and S. I. Temkin, Spectroscopy of Molecular Rotations in Liquids and Gases (Nauka, Novosibirsk, 1982).

${ }^{23}$ M. Loeve, Probability Theory (Van Nostrand, Princeton, 1963).

${ }^{24}$ E. N. Ivanov, Sov. Phys. JETP 18, 1041 (1964); K. A. Valiev and E. N. Ivanov, Sov. Phys. Usp. 16, 1 (1973).

${ }^{25}$ Yu. A. Serebrennikov, Adv. Magn. Opt. Res. 17, 47 (1992).

${ }^{26}$ It has been shown in Ref. 14 that, formally, $\gamma_{\perp}$ could fall outside the region defined by inequality (46), namely $-1 \leqslant y_{\perp} \leqslant 2$. However in both extreme situations: $-1 \leqslant \gamma_{\perp}<0$ and $1<\gamma_{1} \leqslant 2$ the calculated "magnetization" $M_{Z_{L}}^{\text {eff }}(t)$, Eq. (10), can no longer be considered as a good approximation to the physically significant observable, namely the true magnetization given by $\operatorname{Tr}\left(\hat{g} \mathrm{~S}^{\text {eff }}\right)_{Z_{L}}^{(M)} \hat{\rho}^{(M)}(t)$, and it is necessary to consider the time evolution of this quantity.

${ }^{27}$ D. A. Varshalovich, A. N. Moskalev, and V. K. Khersonsky, Quantum Theory of Angular Momentum (World Scientific, Singapore, 1988). 